\title{
Morphological Pattern and Classification of the Superficial Middle Cerebral Vein by Cadaver Dissections: An Embryological Viewpoint
}

\author{
Yasutaka IMADA, ${ }^{1}$ Kaoru KuRISU, ${ }^{2}$ Toru TAKUMI, ${ }^{3,4}$ Hirohiko AOYAMA,,${ }^{5,6}$ \\ Takashi SADATOMO, ${ }^{7}$ Keisuke MigITA, ${ }^{8}$ and Kiyoshi YUKI ${ }^{7}$
}

\author{
${ }^{1}$ Department of Neurosurgery, Yamada Memorial Hospital, Mihara, Hiroshima, Japan; \\ ${ }^{2}$ Department of Neurosurgery, Graduate School of Biomedical and Health Sciences, \\ Hiroshima University, Hiroshima, Hiroshima, Japan; \\ ${ }^{3}$ Department of Integrative Bioscience, Graduate School of Biomedical and Health \\ Sciences, Hiroshima University, Hiroshima, Hiroshima, Japan; \\ ${ }^{4}$ RIKEN Brain Science Institute, Wako, Saitama, Japan; \\ ${ }^{5}$ Department of Anatomy and Developmental Biology, Graduate School of Biomedical \\ and Health Sciences, Hiroshima University, Hiroshima, Hiroshima, Japan; \\ ${ }^{6}$ Faculty of Health Science, Hiroshima International University, \\ Higashihiroshima, Hiroshima, Japan; \\ ${ }^{7}$ Department of Neurosurgery, Higashihiroshima Medical Center, Higashihiroshima, \\ Hiroshima, Japan; \\ ${ }^{8}$ Department of Neurosurgery, Chugoku Rosai Hospital, Kure, Hiroshima, Japan
}

\begin{abstract}
In this study, we used 45 adult cadaveric cerebral hemispheres to investigate the anatomical classification of the superficial middle cerebral vein (SMCV) based on the number of stems, course, and anastomosis at the distal portion. We classified the SMCVs into five types based on embryological concept. Type A (18 cases, $40.0 \%$ ) is that the frontosylvian veins (FSVs) merge with the vein of Trolard (VT) and the vein of Labbé (VL) at the distal portion of the sylvian fissure. Type B (5 cases, 11.1\%) is that the temporosylvian veins (TSVs) merge with the VT and the VL at the distal portion. Type C (13 cases, $28.9 \%$ ) is that no vein merge with the VT and the VL at the distal portion. The VT merges with the SMCV from the FSV and the VL merges with the SMCV from the TSV. They course along the sylvian fissure and merge at the proximal portion. In Type D (eight cases: $17.8 \%$ ), the VT and the VL merge at the distal portion, and the SMCV from the FSV and the SMCV from the TSV join their confluence without merging. Type E (one case, $2.2 \%$ ) show an undeveloped SMCV. Formation rate of intravenous anastomoses or bridging veins(BVs) at the distal portion between the frontosylvian trunk (FST) and the temporosylvian trunk (TST), between the FST and the temporal lobe, and between the TST and the frontal lobe was very low, because these formation may be difficult to occur during the embryological process in which the SMCV is formed from the telencephalic vein.
\end{abstract}

Key words: superficial middle cerebral vein, frontosylvian vein, temporosylvian vein, trans-sylvian approach

\section{Introduction}

Neurosurgery with a pterional approach by frontotemporal craniotomy is obstructed by the superficial middle cerebral vein (SMCV), and its structure and the network of venous return should be considered during

Received November 19, 2018; Accepted March 7, 2019

Copyright $\odot 2019$ by The Japan Neurosurgical Society This work is licensed under a Creative Commons AttributionNonCommercial-NoDerivatives International License. planning of a surgical approach and strategy. Therefore, it is necessary to understand the structure of the SMCV and the anatomical relationships between the frontal lobe, the temporal lobe, and the sylvian fissure. In the past, identification of the SMCV has focused mainly on the outflow point, ${ }^{1-7)}$ however, because the SMCV shows variation in number of stems, course, and anastomoses at the distal portion, it can be difficult to define the SMCV. ${ }^{5,8-15)}$ There is a little detailed discussion of methods to dissect the SMCV in the trans-sylvian approach in the clinical 
literature, and a consensus for a best approach has not been obtained..$^{8,12,14-16)}$

In this study, we examined the morphological pattern and characteristics of the SMCV from detailed observations using cadaveric cerebral hemispheres. Our observations of the number of stems, the course of the SMCV, and distal anastomoses from an embryological viewpoint may establish an anatomical classification of the SMCV that is useful in the trans-sylvian approach. In addition, we investigated how to dissect the SMCV according to this classification.

\section{Materials and Methods}

Superficial middle cerebral veins were examined using $3 \times$ magnification in 45 adult cadaveric cerebral hemispheres in which the arteries and veins had not been perfused with colored silicone. These cadaveric brains were separated from the skull and the dura mater was removed for medical student dissection in 2012. For this reason, we were unable to confirm the final outflow point of the SMCVs, but we could observe the SMCVs on the lateral cerebral surface. We peeled the SMCVs from the surface of the distal to the proximal portion of the sylvian fissure using microscissors and examined the number of stems, course, and anastomoses between adjacent superficial middle cerebral veins, and anastomoses between the SMCV and the frontosylvian veins (FSVs) or temporosylvian veins (TSVs). Although the arteries and veins were not perfused with colored silicone, it was relatively easy to distinguish the arteries and the veins.

\section{Results}

\section{Nomenclature}

The sylvian veins were defined as described in previous studies. ${ }^{5,17,18)}$ The SMCV receive FSVs, PSVs, and TSVs, and usually arise at the posterior end of the sylvian fissure and course anteriorly and inferiorly along the edges of the sylvian fissure. The FSVs are a group of 3-6 veins that descend on the frontal operculum and drain the inferior and adjoining part of the middle frontal gyri and the inferior part of the precentral gyrus. The PSVs are 2-4 small trunks that arise on the postcentral gyrus and the inferior parietal lobule and pass anteroinferiorly toward the sylvian fissure where they join the SMCV. The TSVs are a group of smaller veins that arise along the full extent of the superior temporal gyrus from the temporal pole to the posterior end of the sylvian fissure.

Based on these anatomical characterizations, we tentatively defined the SMCV as follows.
The frontosylvian trunk (FST) is the SMCV that receives FSV or PSV. The temporosylvian trunk (TST) is the SMCV that receives TSV. The superficial middle cerebral common trunk (SMCCT) is the SMCV that receives both FSV and PSV, whose main component is the vein of Trolard, and the TSV, whose main component is the vein of Labbé, at the posterior end of the sylvian fissure (Fig. 1a).

We classified the SMCVs into five types as follows (Fig. 1b). Type A SMCVs correspond to the SMCCT and have some anastomoses with the FST or the FSV on the sylvian fissure. This type was observed in $40.0 \%(n=18)$ of the 45 cerebral hemispheres and was the most frequent form. Anastomoses between the SMCCT and the TSV were observed in $22.2 \%(n=4)$ of these Type A SMCVs. All these anastomoses were small bridging veins between the SMCCT and the temporal lobe, and anastomoses between the SMCCT and the TST was not observed. In one third of Type A cases $(n=6)$, the proximal end of the SMCCT did not empty into any skull base sinus, and became a blind end at the base of the frontal lobe or connected to the deep sylvian veins directly. In two third of Type A cases $(n=12)$, the proximal end of the SMCCT seemed to empty into either skull base sinus according to its course within the observable range, but it was impossible to identify accurately (Table 1).

Type B SMCVs correspond to the SMCCT and have no anastomoses with the FST or the FSV on the sylvian fissure. This type was observed in $11.1 \%$ $(n=5)$ of the 45 cerebral hemispheres. Anastomoses between the SMCCT and the TSV were observed in $60 \%(n=3)$ of these Type B SMCVs. In all cases, the proximal end of the SMCCT seemed to empty into either skull base sinus according to its course within the observable range, but it was impossible to identify accurately (Table 1). The SMCCT was derived from the FST in $78.3 \%$ of cases, while the frequency that the SMCCT was derived from the TST was $21.7 \%$. The frequency of derivation of the SMCCT from the FST was high.

Type C SMCVs show no SMCCT. In other words, the FST and the TST run in parallel along the sylvian fissure and course toward the skull base. This type was observed in $28.9 \%(n=13)$ of the 45 cerebral hemispheres. Anastomoses between the FST and the FSV were observed in $84.6 \%(n=11)$ of these Type C SMCVs, while anastomoses between the TST and the TSV was seen in $53.8 \%(n=7)$. In nine of the 13 cases $(69.2 \%)$, merger of the FST and the TST at the proximal portion was confirmed, but, in the other four cases, it was impossible to identify accurately within the observable range. In all cases, BVs between the FST and the TST were not observed (Table 2). 
a

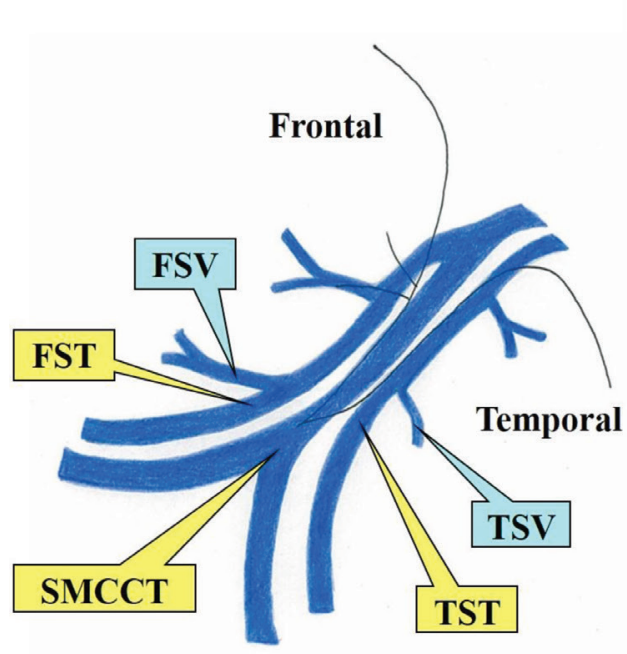

$\mathrm{b}$

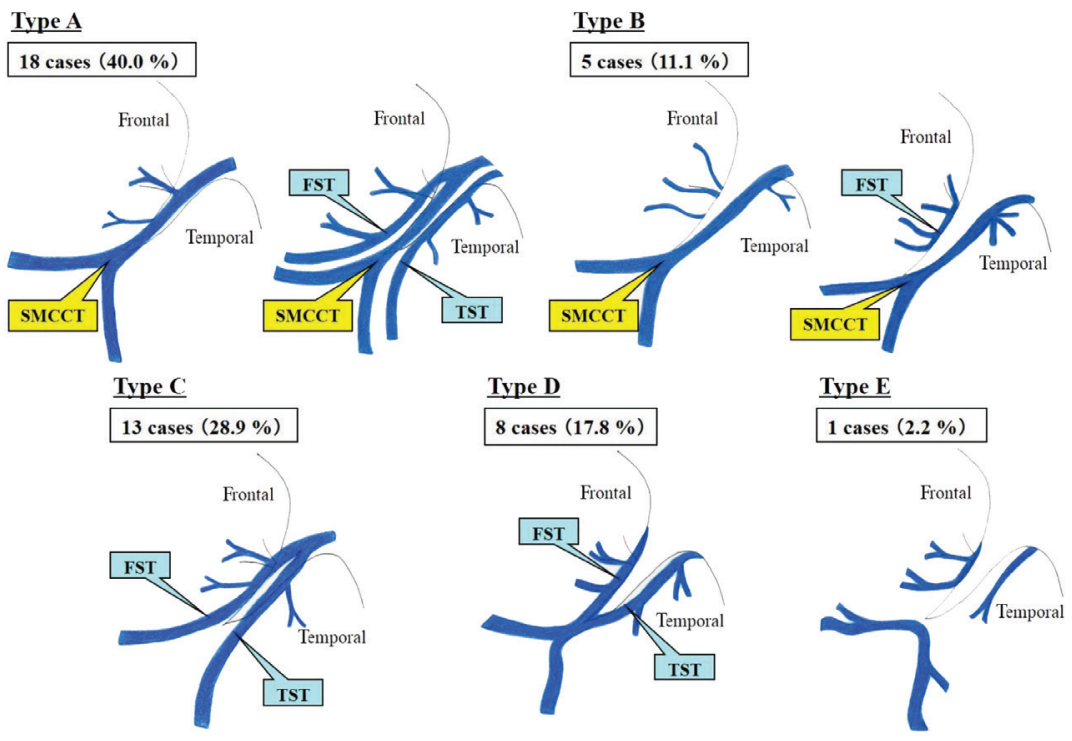

Fig. 1 (a) Nomenclature of the superficial middle cerebral veins. Frontosylvian trunk (FST) is the superficial middle cerebral vein (SMCV) that receives the frontosylvian veins (FSV) or the parietosylvian veins (PSV). Temporosylvian trunk (TST) is the SMCV that receives the temporosylvian veins (TSV). Superficial middle cerebral common trunk (SMCCT) is the SMCV that receives both the FSV (or PSV) and the TSV at the posterior end of the sylvian fissure. (b) Classification of the SMCV.

Table 1 Characteristics of Type A and B SMCV

\begin{tabular}{lccrc}
\hline & $\begin{array}{c}\text { Anastomosis between } \\
\text { the SMCCT and the } \\
\text { temporal side }(\%)\end{array}$ & $\begin{array}{c}\text { Unknown }(\text { dural } \\
\text { sinus? })(\%)\end{array}$ & Blind end (\%) & Deep SV (\%) \\
\cline { 3 - 5 } $\begin{array}{l}\text { Type A }(n=18) \\
\text { Type B }(n=5)\end{array}$ & $22(n=4)$ & $66.6(n=12)$ & $16.7(n=3)$ & $16.7(n=3)$ \\
\hline
\end{tabular}

SMCCT: superficial middle cerebral common trunk, SMCV: superficial middle cerebral vein, SV: sylvian vein, Unknown: unable to evaluate due to specimen damage.

Table 2 Characteristics of Type C and D SMCV

\begin{tabular}{|c|c|c|c|c|c|c|c|}
\hline & \multirow{2}{*}{$\begin{array}{l}\text { Anastomosis } \\
\text { between the } \\
\text { FST and the } \\
\text { FSV }(\%)\end{array}$} & \multirow{2}{*}{$\begin{array}{l}\text { Anastomosis } \\
\text { between the } \\
\text { TST and the } \\
\text { TSV }(\%)\end{array}$} & \multirow{2}{*}{$\begin{array}{l}\text { BV between } \\
\text { the FST } \\
\text { and the } \\
\text { TST }(\%)\end{array}$} & \multirow{2}{*}{$\begin{array}{l}\text { Merger of the } \\
\text { FST and } \\
\text { the TST at } \\
\text { the proximal } \\
\text { portion }(\%)\end{array}$} & \multicolumn{3}{|c|}{ Site of termination of the FST } \\
\hline & & & & & $\begin{array}{c}\text { Unknown } \\
\text { (dural sinus?) } \\
(\%)\end{array}$ & Blind end (\%) & Deep SV (\%) \\
\hline Type C $(n=13)$ & $84.6(n=11)$ & $53.8(n=7)$ & $0(n=0)$ & $69.2(n=9)$ & $100(n=13)$ & $0(n=0)$ & $0(n=0)$ \\
\hline Type D $(n=8)$ & $87.5(n=7)$ & $87.5(n=7)$ & $28.6(n=2)$ & $0(n=0)$ & $12.5(n=1)$ & $62.5(n=5)$ & $25(n=2)$ \\
\hline
\end{tabular}

Merger of the FST and the TST at the proximal portion was unable to be confirmed due to specimen damage in the other four cases of Type C SMCV. BV: bridging vein, FST: fronto-superficial sylvian trunk, SMCV: superficial middle cerebral vein, SV: sylvian vein, TST: temporo-superficial sylvian trunk, Unknown: unable to evaluate due to specimen damage.

Type D SMCVs are a merging of the vein of Trolard and the vein of Labbé at the posterior end of the sylvian fissure. Type D SMCVs divide into the FST and the TST again toward the proximal portion. This type was observed in $17.8 \%(n=8)$ of the 45 cerebral hemispheres. Anastomoses between the FST and the FSV were observed in $87.5 \%$ $(n=7)$ of these Type D SMCVs, while anastomoses between the TST and the TSV were observed in $87.5 \%(n=7)$. Tiny BVs between the FST and the 
TST were observed in 28.6\% $(n=n=2)$ of these Type D SMCVs. In seven of the eight cases of Type D SMCVs, the proximal end of the FST did not empty into any skull base sinus, and was connected to the veins of the basal surface of the frontal lobe or deep sylvian veins directly. The remaining case seemed to empty into either skull base sinus according to its course within the observable range, but it was impossible to identify accurately. In all cases, the proximal end of the TST seemed to empty into either skull base sinus according to its course within the observable range, but it was impossible to identify accurately (Table 2).

Type E SMCVs are underdeveloped on the sylvian fissure. This type was observed in $2.2 \%(n=1)$ of the 45 cerebral hemispheres. In this case, the merger of the vein of Trolard and the vein of Labbé at the posterior end of the sylvian fissure was observed, and BVs between the frontal and the temporal lobe were not observed (Table 3). However, the frontobasal BV was excluded from the evaluation as a $\mathrm{BV}$ in this study.

\section{Discussion}

There have been many morphological classifications of the SMCVs according to draining pattern of veins. ${ }^{3,6)}$ However, we classified into five patterns from an embryological viewpoint, because the process of embryological formation of the SMCV would help to estimate the pattern of the venous return for each type.

From morphological viewpoint, Hacker et al. ${ }^{19}$ classified the superficial veins into three groups according to draining pattern from the cortical surface. The first group, superior cerebral veins, drain the superior part of the medial and lateral surface of the hemisphere, and include the vein of Trolard. These veins empty into the superior sagittal sinus. The second group, the SMCV, drain the regions of the frontal, temporal, and parietal lobes adjoining the sylvian fissure and empty into the sphenoparietal or cavernous sinus. The third group, the inferior cerebral veins, drain the lateral surface of the temporal lobe and the basal surface of the temporal and occipital lobes, and include the vein of Labbé. These veins empty into the transverse or tentorial sinuses.

Table 3 Characteristics of Type E SMCV

BV between the frontal lobe and the temporal lobe (\%)

\begin{tabular}{lc}
\hline Type $\mathrm{E}(n=1)$ & $0(n=0)$ \\
\hline BV: bridging vein, SMCV: superficial middle cerebral vein.
\end{tabular}

These superficial veins are formed from the development of the pial venous network originating from the meninx primitiva. ${ }^{20-22)}$ According to Padget, the superficial telencephalic vein drains the lateral surface of the hemisphere and the deep telencephalic vein drains the sylvian fossa in early embryonic stages. The former corresponds to the subsequent SMCV, and the latter corresponds to the subsequent deep middle cerebral vein. These veins converge on the sylvian fossa forward and downward, and empty into the transverse sinus via the primitive tentorial sinus. Subsequent growth of the posterior cerebral hemisphere causes the tentorial sinus to elongate posteriorly, and the anterior portion is shifted medially to anastomose with the sphenoparietal or cavernous sinus. The posterior part of the primitive tentorial sinus regresses gradually and the anastomoses between the sphenoparietal and cavernous sinuses develop. ${ }^{21,23)}$ In accordance with the completeness of these two secondary anastomoses, the variation of the outflow of the superficial telencephalic vein occurs. ${ }^{2,6,21,23)}$ In the distal part, the SMCV and its tributaries are well developed from the superficial telencephalic vein and the vein around the sylvian fissure at the gestational ages of 3 and 4 months. The SMCV and its tributaries rapidly regress from the 5th to 7th month. The superior and inferior cerebral veins, on the other hand, develop rapidly during this period. The anastomotic veins between the SMCV, the superior cerebral vein and the inferior cerebral vein are also recognizable during this period. ${ }^{21,23,24)}$ This process of embryological formation of the SMCV shows individual differences in the degree of degeneration of the SMCV and in the degree of the formation of the anastomoses between the SMCV and the superior or inferior cerebral vein. This variation results in the various patterns of the distal portion of the SMCV (Fig. 2a). For this reason, a classification based on the variation of outflow path of the SMCV has been reported, but there are few studies examining the number of stems, course, and anastomoses of the SMCV at the distal portion. ${ }^{8,12,14,15)}$ So we investigated the anatomical classification of the SMCV based on the number of stems, course, and the anastomotic form at the distal portion from an embryological viewpoint.

The SMCV is composed of the three components: the FSV, which drains the lower part of the lateral frontal lobe; the PSV, which drains the postcentral gyrus and the inferior parietal lobule; and the TSV, which drains the superior temporal gyrus from the temporal pole to the posterior end of the sylvian fissure. ${ }^{5,18)}$ In embryonic development, the SMCV is formed by joining the telencephalic veins draining each region of the sylvian fissure. 
A

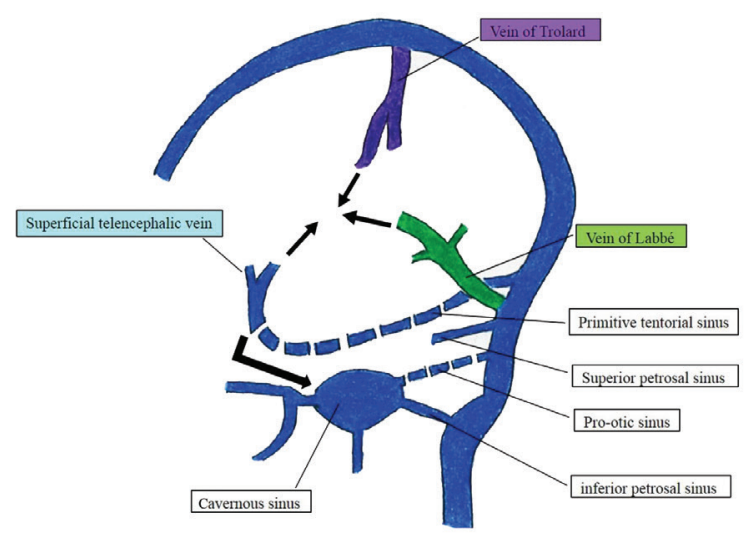

B Type A

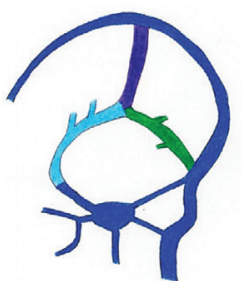

Type B

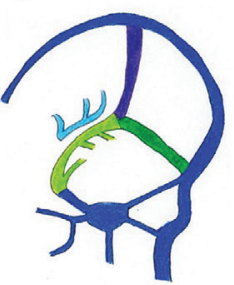

Type A

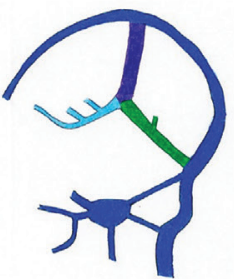

Type C

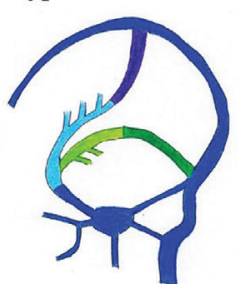

Type A

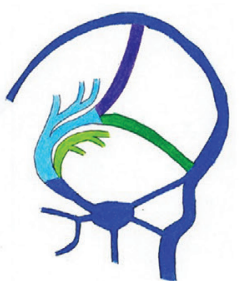

Type D

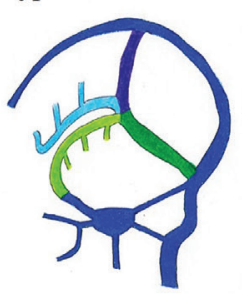

Type B

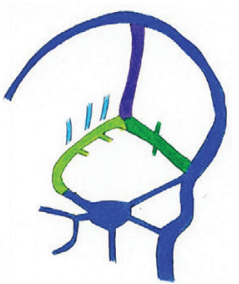

Type E

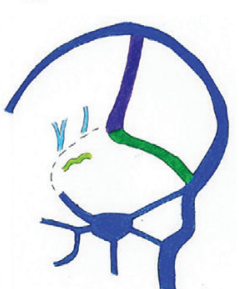

Fig. 2 (a) Embryologic process of the superficial middle cerebral vein (SMCV). (b) Consideration on the origin of the SMCV from the viewpoint of embryologic development. Purple: vein of Trolard, light blue: frontosylvian trunk or frontosylvian vein, green: vein of Labbé, light green: temporosylvian trunk or temporosylvian vein.

There are four major points along with an embryological viewpoint to describe our classification obtained from detail morphological observation of 45 adult cadaveric cerebral hemispheres.

(1) Flow of the SMCV should be consistent with an embryologic development (Fig. 2b).

Type A SMCV can be described as superior cerebral veins, composed mainly of the vein of Trolard, and the inferior cerebral veins, composed mainly of the vein of Labbé, merged with the FST derived from the FSV at the distal portion of the sylvian fissure.

Type B SMCV can be described as superior cerebral veins, composed mainly of the vein of Trolard, and the inferior cerebral veins, composed mainly of the vein of Labbé, that merge with the TST derived from the TSV at the distal portion of the sylvian fissure.

Type C SMCV can be described as superior cerebral veins, composed mainly of the vein of Trolard, and the inferior cerebral veins, composed mainly of the vein of Labbé, that did not merge at the distal portion of the sylvian fissure. The former merged with the FST and the latter with the TST at the distal portion of the sylvian fissure, and they ran in parallel along the sylvian fissure and merge at the proximal portion.

Type D SMCV can be described as superior cerebral veins, composed mainly of the vein of Trolard, and the inferior cerebral veins, composed mainly of the vein of Labbé, that merge at the distal portion of the sylvian fissure. The FST and the TST join to their confluence, and the FST and the TST do not merge at the proximal portion.

Type E SMCV can be described as an underdeveloped SCMV regardless of whether superior cerebral veins, composed mainly of the vein of Trolard, and the inferior cerebral veins, composed mainly of the vein of Labbé, merge or not.

(2) Flow of the BVs of the SMCV should be along with an embryological development. Our results demonstrate that the rate of anastomoses or BVs between the FST and the TST at the distal portion of the sylvian fissure was very low, the rate of BVs between the FST and the temporal lobe was very low, and the rate of BVs between the TST and the frontal lobe was also very low. These results indicate that the formation of intravenous anastomoses or BVs between the FST and the TST, the FST and the temporal lobe, and the TST and the frontal lobe at the distal portion of the sylvian fissure may be difficult to occur during the embryological process in which the FST and the TST are formed from the superficial telencephalic vein according to each drainage area.

(3) The venous return of the SMCV should be revealed as development pattern. Although we could not evaluate accurately the outflow point of the SMCV in this study, some of the FST or the SMCCT derived from the FSV did not empty into the skull base sinuses, whereas all of the TST and the SMCCT derived from the TSV emptied into the skull base sinuses. Thus, the sphenobasal and sphenopetrosal types defined by Hacker, or the emissary type, superior petrosal type, and basal type defined by Suzuki may tend to be formed from the TST or the SMCCT derived from the TSV., ${ }^{3,6)}$ And, in the case which the SMCCT or the FST becomes blind end at the base of the frontal lobe, the venous return may flow from the proximal portion to the 
distal portion. Further study is required to clarify these points.

(4) Intraoperative dissection of the SMCV in a trans-sylvian approach should be performed based on these anatomical features. Although many neurosurgeons start to dissect between the SMCV and the frontal lobe in a trans-sylvian approach, it is often necessary to cut off the BVs with the frontal lobe in order to expand the operative field widely, which may cause vein infarction. On the other hand, some reports recommend dissection between multiple superficial middle cerebral veins or between the SMCV and the temporal lobe based on the number of stems, course and anastomotic form, ${ }^{12,14)}$ but there is no consensus yet.

For cerebrovascular surgery, we strongly suggest dissection of the SMCV based on our classification to preserve the venous structure (Fig. 3). In Type A SMCV, it is recommended to start to dissect the SMCV on the temporal side of the SMCCT and then dissect between the SMCCT or the FST and the frontal lobe on the base side as much as needed. These procedures will make it possible to increase the mobility of the temporal lobe and get wide operative field while preserving veins. Of course, it is necessary to dissect the frontobasal $\mathrm{BV}$ from the frontal lobe as much as possible to increase the more mobility of the frontal lobe. On the other hand, it may be enough to dissect only the temporal side of the SMCCT for some cases of an aneurysm of middle cerebral artery's bifurcation in distal trans-sylvian approach. In Type B SMCV, it is recommended to dissect the SMCV on the frontal side of the SMCCT. In Type C SMCV, it is recommended to start to dissect between the FST and the TST until their proximal junction and then dissect between the FST and the frontal lobe on the base side as much as needed. These procedures will make it possible to increase the mobility of the temporal lobe and get wide operative field while preserving veins. It may be enough to dissect only between the FST and the TST until their proximal junction for some cases of an aneurysm of middle cerebral artery's bifurcation in distal trans-sylvian approach. In Type D SMCV, it is recommended to start to dissect between the FST and the TST, and it is unnecessary to dissect between the FST and the frontal lobe, if the proximal end of the FST is connected to the veins of the basal surface of the frontal lobe or deep sylvian veins directly. In Type E SMCV, there is no choice but to dissect between the frontal and the temporal lobe. And, in the case which the SMCCT or the FST becomes blind end at the base of the frontal lobe in Type A or D SMCV, the sacrifice of the SMCCT or the FST may cause the venous infarction of the frontal lobe with a high probability. Because the SMCCT or the FST may drain the lower part of the lateral frontal

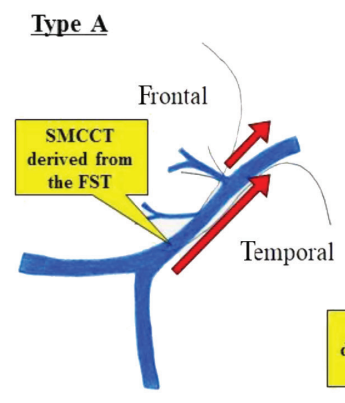

Type C

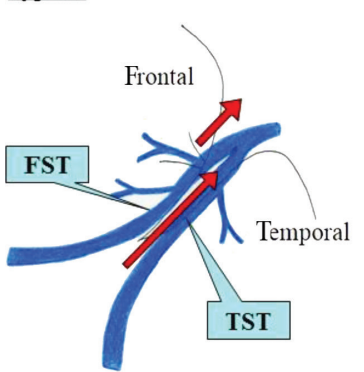

Type D

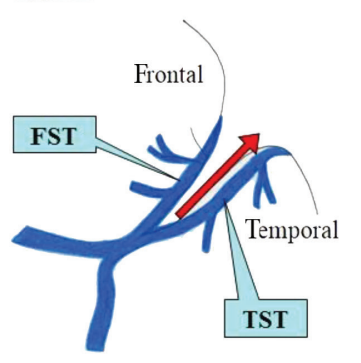

Type B

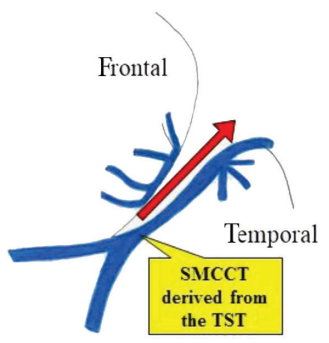

Type $\mathbf{E}$

Fig. 3 Dissection of the superficial middle cerebral vein (SMCV) based on our classification to preserve the venous structure. Red arrows show the dissection line of the SMCV to preserve the veins as much as possible. FST: frontosylvian trunk, SMCV: superficial middle cerebral vein, TST: temporosylvian trunk, SMCCT: superficial middle cerebral common trunk. 
lobe mainly from the proximal portion to the distal portion. Although it is necessary to investigate in more detail by clinical cases, this classification of the SMCVs with the above characteristics can be helpful in discussing how to dissect the SMCV in a trans-sylvian approach.

\section{Conclusion}

The SMCVs show various courses and anastomoses. We classified them into five types from an embryological viewpoint. To preserve these veins during surgery, it is useful to dissect the SMCV according to these classifications in trans-sylvian approach.

\section{Conflicts of Interest Disclosure}

The authors report no conflict of interest concerning the materials or methods used in this study or the findings specified in this paper.

\section{References}

1) Bisaria KK: The superficial sylvian vein in humans: with special reference to its termination. Anat Rec 212: 319-325, 1985

2) Chung JI, Weon YC: Anatomic variations of the superficial middle cerebral vein: embryologic aspects of the regressed embryonic tentorial sinus. Interv Neuroradiol 11: 115-122, 2005

3) Hacker H: [Outflow pathways of the sylvian vein group]. Radiologe 8: 383-387, 1968 (German)

4) Kageyama Y, Fukuda K, Kobayashi S, et al.: Cerebral vein disorders and postoperative brain damage associated with the pterional approach in aneurysm surgery. Neurol Med Chir (Tokyo) 32: 733-738, 1992

5) Oka K, Rhoton AL Jr, Barry M, Rodriguez R: Microsurgical anatomy of the superficial veins of the cerebrum. Neurosurgery 17: 711-748, 1985

6) Suzuki Y, Matsumoto K: Variations of the superficial middle cerebral vein: classification using threedimensional CT angiography. AJNR Am J Neuroradiol 21: 932-938, 2000

7) Wolf BS, Huang YP, Newman CM: The superficial sylvian venous drainage system. Am J Roentgenol Radium Ther Nucl Med 89: 398-410, 1963

8) Aydin IH, Kadioğlu HH, Tüzün Y, Kayaoğlu CR, Takçi E: The variations of sylvian veins and cisterns in anterior circulation aneurysms. An operative study. Acta Neurochir (Wien) 138: 1380-1385, 1996

9) Aydin IH, Tüzün Y, Takçi E, Kadioğlu HH, Kayaoğlu CR, Barlas E: The anatomical variations of sylvian veins and cisterns. Minim Invasive Neurosurg 40: 68-73, 1997

10) Browder J, Krieger AJ, Kaplan HA: Cerebral veins in the surgical exposure of the middle cerebral artery. Surg Neurol 2: 359-363, 1974

11) Ito Z: Microsurgery of Cerebral Aneurysm. Tokyo: Nishimura, Elsevier, 1985, pp. 95-201

12) Kazumata K, Kamiyama H, Ishikawa T, et al.: Operative anatomy and classification of the sylvian veins for the distal transsylvian approach. Neurol Med Chir (Tokyo) 43: 427-433; discussion 434, 2003

13) Ono M, Rhoton AL, Peace D, Rodriguez RJ: Microsurgical anatomy of the deep venous system of the brain. Neurosurgery 15: 621-657, 1984

14) Sakata Y, Hadeishi H, Tanaka M, et al.: Strategy of superficial sylvian vein dissection on distal sylvian approach. Inspection of microsurgical anatomy. Surg Cereb Stroke 38: 387-390, 2010

15) Yasargil MG: Microneurosurgery. Stuttgart: Georg Thieme Verlag, Vol I, 1984, pp. 5-168

16) Kazumata K, Yokoyama Y, Sugiyama T, et al.: Anatomic consideration for trans-sylvian approach. Jpn J Neurosurg 21: 680-687, 2012

17) Gibo H, Carver CC, Rhoton AL Jr, Lenkey C, Mitchell RJ: Microsurgical anatomy of the middle cerebral artery. J Neurosurg 54: 151-169, 1981

18) Rhoton AL Jr: Rhoton's Cranial Anatomy and Surgical Approaches: Philadelphia, Lippincott Williams \& Wilkins, 2003, pp. 187-234

19) Hacker H: Normal supratentorial veins and dural sinus. In Newton TH, Potts DG (eds): Radiology of the Skull and Brain, St. Louis: C.V. Mosby, 1974, pp. 1851-1877

20) Streeter GL: The developmental alterations in the vascular system of the brain of the human embryo. Contrib Embryol 8: 5-38, 1918

21) Padget DH: The development of the cranial venous system in man, from the viewpoint of comparative anatomy. Contrib Embryol 36: 79-140, 1957

22) O'Rahilly R, Müller F: The meninges in human development. J Neuropathol Exp Neurol 45: 588-608, 1986

23) Padget DH: The cranial venous system in man in reference to development, adult configuration, and relation to the arteries. Am J Anat 98: 307-355, 1956

24) Okudera T, Ohta T, Huang YP, Yokota A: Developmental and radiological anatomy of the superficial cerebral convexity vessels in the human fetus. J Neuroradiol 15: 205-224, 1988

Address reprint requests to: Yasutaka Imada, MD, Department of Neurosurgery, Yamada Memorial Hospital, 6-2-1 Miyaura, Mihara, Hiroshima 723-0051, Japan. e-mail: yasutaka5682@yahoo.co.jp 